\title{
The right hemisphere in esthetic perception
}

\section{Bianca Bromberger, Rebecca Sternschein, Page Widick, William Smith II and Anjan Chatterjee*}

Department of Neurology, The University of Pennsylvania, Philadelphia, PA, USA

\section{Edited by:}

Idan Segev, The Hebrew University of Jerusalem, Israel

\section{Reviewed by:}

Juliana Yordanova, Bulgarian Academy of Sciences, Bulgaria

Bernd Weber,

Rheinische-Friedrich-Wilhelms

Universität, Germany

\section{*Correspondence:}

Anjan Chatterjee, Department of Neurology, The University of

Pennsylvania, 3 West Gates, 3400

Spryce Street, Philadelphia, PA

19104, USA.

e-mail:anjan@mail.med.upenn.edu
Little about the neuropsychology of art perception and evaluation is known. Most neuropsychological approaches to art have focused on art production and have been anecdotal and qualitative. The field is in desperate need of quantitative methods if it is to advance. Here, we combine a quantitative approach to the assessment of art with modern voxel-lesionsymptom-mapping methods to determine brain-behavior relationships in art perception. We hypothesized that perception of different attributes of art are likely to be disrupted by damage to different regions of the brain. Twenty participants with right hemisphere damage were given the Assessment of Art Attributes, which is designed to quantify judgments of descriptive attributes of visual art. Each participant rated 24 paintings on 6 conceptual attributes (depictive accuracy, abstractness, emotion, symbolism, realism, and animacy) and 6 perceptual attributes (depth, color temperature, color saturation, balance, stroke, and simplicity) and their interest in and preference for these paintings. Deviation scores were obtained for each brain-damaged participant for each attribute based on correlations with group average ratings from 30 age-matched healthy participants. Right hemisphere damage affected participants' judgments of abstractness, accuracy, and stroke quality. Damage to areas within different parts of the frontal parietal and lateral temporal cortices produced deviation in judgments in four of six conceptual attributes (abstractness, symbolism, realism, and animacy). Of the formal attributes, only depth was affected by inferior prefrontal damage. No areas of brain damage were associated with deviations in interestingness or preference judgments. The perception of conceptual and formal attributes in artwork may in part dissociate from each other and from evaluative judgments. More generally, this approach demonstrates the feasibility of quantitative approaches to the neuropsychology of art.

\section{Keywords: aesthetics, brain damage, neuropsychology, neuroesthetics}

\section{INTRODUCTION}

Historically, art and esthetics have been well ensconced in the humanities and have not been considered seriously within the sciences. Fechner (1876) began the field of empirical esthetics. More than a century later, neuroscience is playing catch-up, and is finally coming of age (Skov and Vartanian, 2009; Chatterjee, 2011). Theoretical positions and a few books linking neuroscience to art have appeared (Ramachandran and Hirstein, 1999; Zeki, 1999; Livingstone, 2002; Chatterjee, 2004a). Empirical studies using imaging techniques looking at our responses to beauty (Aharon et al., 2001; Ishai, 2007; Winston et al., 2007; Chatterjee et al., 2009) as well as to different kinds of artwork (Kawabata and Zeki, 2004; Vartanian and Goel, 2004; Jacobsen et al., 2005; Ishai et al., 2007; Cela-Conde et al., 2009) are being published. Recent conferences devoted to art and neuroscience (Nadal and Pearce, 2011) attest to the growing interest in the biology of esthetics. In this paper, we examine the state of one important aspect of neuroesthetics, the neuropsychology of art (Chatterjee, 2004b; Bogousslavsky and Boller, 2005; Zaidel, 2005). We outline reasons that this aspect of neuroesthetics has been relatively undeveloped and report our initial attempts to rectify this situation.

Since the late nineteenth century, much of our knowledge of the brain bases of cognitive and affective functions has been derived from observations of people with brain damage. From close clinical observations made by Broca, Wernicke, Lichtheim, Lissaur, and Leipmann, the basic tenets of the biology of language, visual semantics, and motor control were established. Over the twentieth century, cognitive neurology and neuropsychology benefited from methods of experimental psychology. Our understanding of memory, emotional processing, decision-making and virtually every domain of cognition advanced from analysis of patients with brain damage. Despite the recent ascendency of functional neuroimaging, the inferential power of lesion studies is robust (Chatterjee, 2005; Fellows et al., 2006). Yet, relatively little about the neuropsychology of art is known.

Many have made observations of the kind of art produced by people with neurologic disease (Bogousslavsky and Boller, 2005; Zaidel, 2005). These observations are made with the hope of inferring the neural bases for artistic production from its derangement by brain damage (Chatterjee, 2006). Despite the fact that such observations date back at least to the 1940s (Alajouanine, 1948), the field has not matured (Chatterjee, 2009). Artists with brain damage that continue to produce a body of work are rare, and it is difficult, if not impossible, to conduct large-scale group studies of artistic production. Most reports describe anecdotal observations and offer a few art examples from which inferences are drawn. 
Thus, we are left with a collection of anecdotes that are fascinating by themselves, but do not contribute to a comprehensive understanding of the systems involved, or any formal tests of hypotheses. A critical obstacle to advancing this work is the lack of quantitative measures. How do we quantify a work of art? Doing so must be critical if we are to measure change. How can we assess change if we do not know what is changing and can reliably measure this change?

To address this issue of how to measure change in artwork, we developed a test called the assessment of art attributes (AAA; Chatterjee et al., 2010). We designed the AAA keeping in mind the need for componential analysis and quantification in the neuropsychology of art. The AAA is based on the widely held assumption that artworks have formal-perceptual qualities and content-conceptual qualities (Russell and George, 1990; Woods, 1991). We selected six formal-perceptual attributes and six content-conceptual attributes based on a review of the literature with special consideration to the kinds of attributes thought to have changed in individuals with brain damage. The formal-perceptual attributes correspond to early and intermediate visual processing. They are: Color temperature (warm-cold), Color saturation (calm-vibrant), Stroke style (controlled-loose), Depth (flat-deep), Balance (low-high), and Complexity (simple-complex). The content-conceptual attributes correspond to higher/late visual processing and its contact with other domains, like semantics and emotional systems. They are: Representational accuracy (less-more), Abstractness (lessmore), Realism (less-more), Animacy (less-more), Symbolism (less-more), and Emotionality (less-more). We familiarize each participant on each attribute. Their assessments are made using a Likert scale, giving quantitative form to these descriptive attributes. The paintings in the AAA were selected from the Western canon, covering different time periods. A well-known artist created each painting to ensure reasonable esthetic quality in our stimuli. However, the selected paintings were not the artists' most popular works (e.g., Hopper's Nighthawks) that might be familiar to even artistically naïve participants.

We have shown that the AAA can be used to assess systematic change in the art produced by people with neurological disease. Using the AAA (Smith et al., 2011), we reported that in patients with left or right focal brain damage, art becomes more abstract, distorted, and less realistic. The paintings are also produced with looser strokes, less depth, and more vibrant colors. Notably, art produced following left brain damage, becomes more symbolic, a change not seen in right brain damage. By contrast, the paintings of people with Alzheimer's Disease became more abstract and symbolic and less realistic and depictively accurate (van Buren et al., 2010).

If our understanding of the nature of artistic production following brain damage has been rudimentary, our knowledge of the effects of brain damage on artistic perception is virtually nonexistent. Based on extant neuropsychological (Chatterjee, 2004b) and functional neuroimaging (Brown et al., 2011) observations, it is unlikely that we evolved perceptual and semantic representations and emotional neural systems designed uniquely for esthetic experiences. Rather, particular combination of regional activations dedicated to general perceptions and emotions give rise to esthetic experiences. The experience of looking at and appreciating visual art likely relies on a diverse set of perceptual and cognitive processes (Chatterjee, 2004a; Leder et al., 2004; Nadal et al., 2008). From admiring the precision of a portrait to responding to the emotional resonance of a landscape, art requires the viewer to perceive many attributes while also forming judgments of liking and interest. We know little about the areas of the brain that are responsible for the perception and evaluation of visual art. The question of how to adequately quantify deviations in perception applies in the same way that it does to deviations in production. Here, we show that the AAA can be used to assess brain-behavior relationships in art perception.

We focus our investigation on the role of the right hemisphere. The right hemisphere participates prominently in visual spatial attention and representation (Heilman et al., 1993; Chatterjee, 2003). Despite limited evidence for the popular view, the right hemisphere is often considered the artistic hemisphere. For our initial attempts to investigate the neural correlates of art perception, we chose to focus on right hemisphere damage patients to avoid confounding language comprehension with judgment in our study. For example, if a participant does not understand what the word "symbolic" means, it would be difficult to assess their judgment of symbolism in any painting. We limited our investigation to the perceptual abilities of artistically inexperienced or "naïve" brain-damaged participants, given that artistically experienced individuals may judge art differently (Cupchik and Gebotys, 1988; Hekkert and Van Wieringen, 1996; Chatterjee et al., 2010). In principle, the same study could be conducted in patients with expertise in art. In practice, such patients are less common in our population. Finally, we use contemporary lesion analysis methods in our study. Voxel-lesion-symptom mapping (VLSM) techniques allow us to formally assess the way in which damage to a brain area correlates with behavioral scores (Bates et al., 2003; Kimberg et al., 2007; Wu et al., 2007), with the advantage that one does not have to establish a deficit cut-off. Rather, behavior in VLSM is considered a continuous variable.

To summarize, neuropsychology has historically been an important aspect of cognitive neuroscience. Yet, the neuropsychology of art has been relatively underdeveloped. In our view, an important reason for this lack of development has been the lack of quantitative methods. To rectify this problem, we developed the AAA to quantify artistic attributes. We also use modern lesion analyses techniques to establish brain-behavior relationships.

\section{MATERIALS AND METHODS PARTICIPANTS}

Twenty individuals with damage to their right hemisphere from stroke (mean age 58.7, 5 men, 15 women) and 30 age-matched healthy controls (mean age 58.8, 9 men, 21 women) participated in the study. Subjects were recruited from the Focal Brain Lesion Database at the Center for Cognitive Neuroscience at the University of Pennsylvania.

\section{PRE-TEST SCREENING}

Participants with brain damage were given a set of visual tests including a shape detection test, a dot counting test, and a position discrimination test from the visual object and spatial perception battery (VOSP; Warrington and James, 1991), as well an Ishihara 
test for colorblindness and a Grayscales test (Mattingley et al., 2004) for right-left bias. They were also given basic background neuropsychological screening tests (see Table $\mathbf{1}$ ).

Participants completed a questionnaire that indicated their experience with visual art. Assessors included the number of art and art history classes taken, frequency of museum and gallery visits, and time spent making or reading about visual art. Based on previous data (Chatterjee et al., 2010) only participants with a score of less than 14 were deemed artistically naïve and were included in the study. Four patients did not meet this criterion and were not included in the analysis reported here.

\section{ASSESSMENT OF ART ATTRIBUTES SCALE}

We used the AAA battery to obtain a quantitative measure of individuals' abilities to judge perceptual and conceptual qualities of visual art. The AAA measures one's ability to perceive 12 different attributes ( 6 formal and 6 conceptual) of visual art (Chatterjee et al., 2010). Participants rate 24 images of paintings from the Western art historical canon (Table 2) on each of the 12 scales on a 5-point Likert scale. Before beginning the AAA battery, participants look at each image to orient themselves to the range of styles of paintings they would be rating. In order to define the dimensions of each attribute scale, participants first see a training slide and two example images that illustrate the extremes of the scale. Participants are allowed to ask clarification questions before proceeding. Participants then rate each of the images of paintings on a 5-point Likert scale. Images are presented in random order and no time limit is imposed. After all images have been rated on the 12 formal and conceptual qualities, participants then evaluate the paintings. They rate each painting for preference and for interest on a 5 -point Likert scale.

\section{LESION DATA}

Every patient's lesion was drawn on a standard brain template ("Colin 27" from the MNI) by one of two senior neurologists. Using MRICron, lesions are defined with respect to anatomically defined structures (e.g., inferior frontal gyrus, angular gyrus, etc.) as well as Brodmann areas using Automated Anatomical Labeling and Brodmann Areas maps available in the MRICro software package (Rorden and Karnath, 2004). VLSM correlations were assessed by regressing behavioral scores on lesion status scores across subjects independently for each voxel. Only voxels that included at least two participants with brain damage analyzed using a false discovery rate of 0.01 (Figure 1).

\section{ANALYSIS AND RESULTS}

The group results of the screening tasks are shown in Table 1. In general patients did well on these tasks. Data from age and artexperience matched control participants were used to develop a baseline measure of esthetic perception and evaluation. For each scale, the 24 paintings were assigned a unique rank order based on their average rating by control participants. Then, individual

Table 1 | Patient demographics and screening data.

\begin{tabular}{|c|c|c|c|c|c|c|c|c|c|c|}
\hline Pt\# & Age & Sex & Years Ed & AMNART & $\begin{array}{l}\text { WAIS- } \\
\text { III }\end{array}$ & $\begin{array}{l}\text { Shape detection } \\
\text { (\% Acc) }\end{array}$ & $\begin{array}{l}\text { Dot counting } \\
\text { (\% Acc) }\end{array}$ & $\begin{array}{l}\text { Position discrimina- } \\
\text { tion ( } \% \text { Acc) }\end{array}$ & Grayscales* & $\begin{array}{l}\text { Ishihara test for } \\
\text { colorblindness } \\
\text { (\% Acc) }\end{array}$ \\
\hline 01 & 69.6 & $M$ & 12 & 122 & 16 & - & - & - & - & - \\
\hline 02 & 68.4 & $\mathrm{~F}$ & 16 & 113 & 10 & 100 & 100 & 95 & -0.19 & 87.5 \\
\hline 03 & 55.0 & $\mathrm{~F}$ & 18 & 121 & 11 & 100 & 100 & 100 & 0.00 & 100 \\
\hline 04 & 45.1 & $\mathrm{~F}$ & 16 & 119 & 12 & 85 & 100 & 100 & -0.50 & 100 \\
\hline 05 & 69.4 & $\mathrm{~F}$ & 12 & 110 & 12 & 90 & 80 & 100 & -0.25 & 100 \\
\hline 06 & 80.8 & $\mathrm{~F}$ & 18 & 115 & 14 & 95 & 100 & 100 & 0.56 & 93.75 \\
\hline 07 & 74.7 & $M$ & 12 & - & - & 80 & 60 & 65 & 0.94 & 56.25 \\
\hline 08 & 59.4 & $\mathrm{~F}$ & 14 & 117 & 11 & 100 & 100 & 95 & -0.19 & 93.75 \\
\hline 09 & 61.1 & $\mathrm{~F}$ & 16 & - & - & - & - & - & - & - \\
\hline 10 & 55.1 & $M$ & 12 & 112 & 10 & 100 & 100 & 100 & 0.19 & 87.5 \\
\hline 11 & 53.2 & $M$ & 10 & - & - & 100 & 70 & 90 & 0.19 & 100 \\
\hline 12 & 58.1 & $\mathrm{~F}$ & 16 & - & - & 100 & 90 & 100 & -0.19 & 100 \\
\hline 13 & 77.2 & $\mathrm{~F}$ & 12 & 99 & - & 80 & 100 & 90 & 0.56 & 100 \\
\hline 14 & 48.6 & $\mathrm{~F}$ & 11 & 89 & 7 & 100 & 100 & 100 & -0.19 & 100 \\
\hline 15 & 69.6 & $\mathrm{~F}$ & 18 & 125 & 14 & 95 & 100 & 100 & -0.25 & 100 \\
\hline 16 & 65.0 & $M$ & 11 & 97 & 8 & 100 & 90 & 75 & -0.88 & 81.25 \\
\hline 17 & 41.3 & $\mathrm{~F}$ & 12 & 110 & 10 & 100 & 90 & 100 & 0.50 & 100 \\
\hline 18 & 46.8 & $F$ & 12 & - & 6 & 100 & 70 & 75 & 1.00 & 100 \\
\hline 19 & 49.9 & $\mathrm{~F}$ & 12 & 106 & 4 & 100 & 100 & 100 & -0.06 & 100 \\
\hline 20 & 33.0 & $\mathrm{~F}$ & 12 & 106 & - & - & - & - & - & - \\
\hline Avg & 59.07 & & 13.60 & 110.73 & 10.36 & 95.59 & 91.18 & 93.24 & 0.07 & 94.12 \\
\hline SD & 12.77 & & 2.62 & 10.05 & 3.30 & 7.26 & 13.17 & 11.03 & 0.50 & 11.37 \\
\hline
\end{tabular}

*Grayscales test for left-right bias $(+1=$ rightward bias, $-1=$ leftward bias). 
control participants' scores were correlated with the rank order for each scale using Spearman's Rho, as a measure of non-parametric correlation. If an individual's Rho statistic fell two SD below the average Rho on a particular scale, their ratings were not used in establishing the rank order of paintings for that attribute. See Chatterjee et al. (2010) for details of these procedures.

Table 2 | List of paintings used in the AAA.

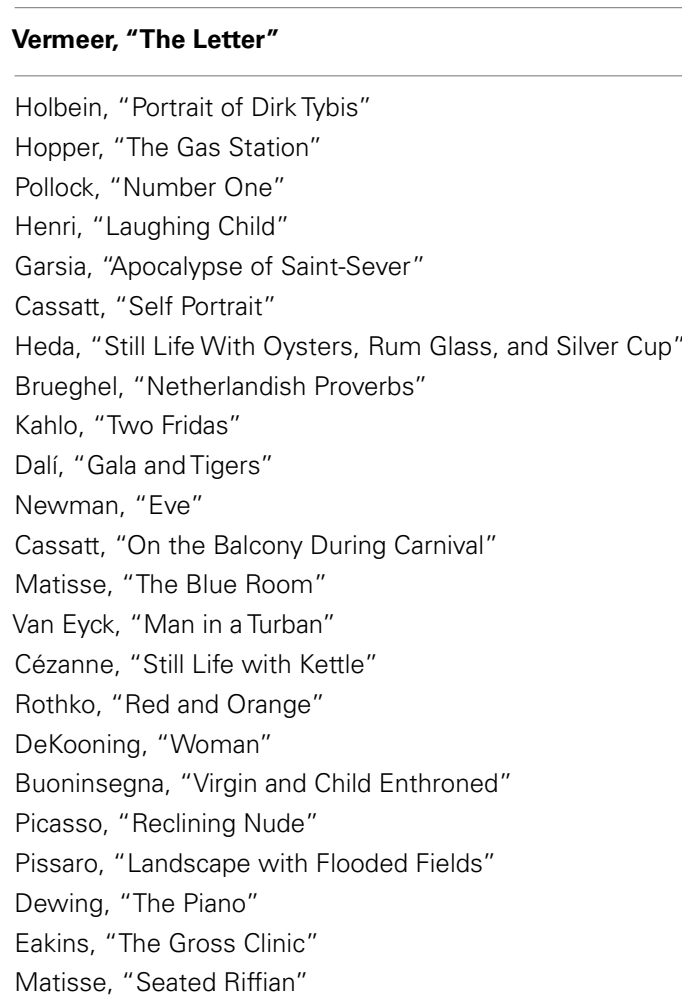

The results of this analysis demonstrate the reliability of the AAA. Controls tended to have high average Spearman's Rho and low SE for each of the 12 attribute scales and interest and preference ratings (Table 3 ).

Once the rank order of paintings was established for each of the 12 scales, we determined the degree to which right hemisphere damaged individuals' esthetic perceptual abilities deviated from normal. Using Spearman's Rho, we correlated each braindamaged individual's ratings on each attribute with the rank order determined by the group control data. Then, for each attribute, the individual's Rho was subtracted from the average Rho of the controls for a difference score. This difference score reflects the degree to which a brain-damaged participant's ratings of a particular attribute differed from the average ratings of the normal control participants (Table 3 ).

We were interested in querying specific possible relationships between our screening tasks and the patients' judgments of different attributes. Specifically, could color perception as measured by the performance of the Ishihara plates account for deviations in judgment of either hue or tone? These correlations were not significant. Similarly, could performance on low-level perceptual tests from the VSOP account for performance on other perceptual judgments. We found a correlation between performance on shape detection and deviations on the attribute of simplicity $(r=0.51$, $p<0.05)$.

To establish brain-behavior correlations of esthetic judgment and evaluation, two analyses were conducted. The first analysis investigated whether right hemisphere damage in general produced specific impairments in esthetic impairment. The second analysis tested for more specific locations within the right hemisphere that were likely to produce impairments in esthetic perception. We should be clear that the results of the first analysis are not predictive of the second. For example, as a group, the brain-damaged individuals might be at floor performance on a

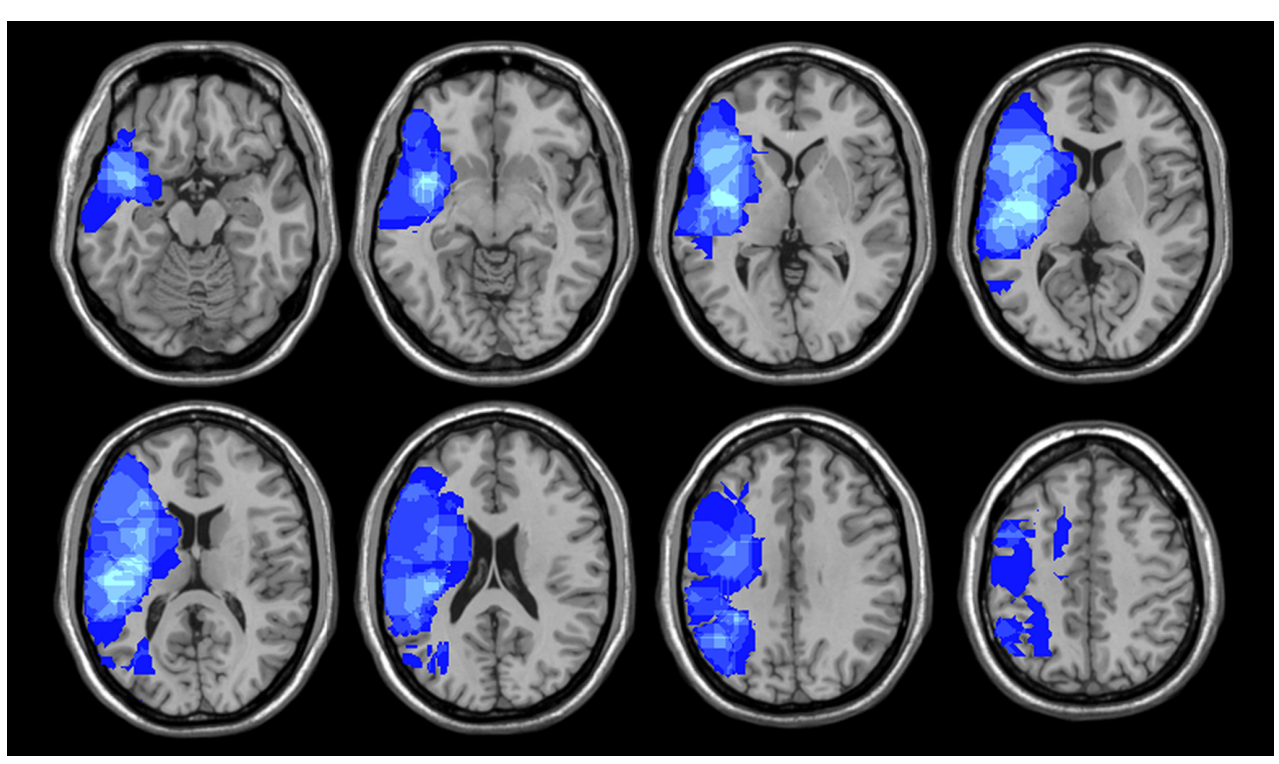

FIGURE 1 | Lesion coverage map within the right hemisphere. 
Table 3 | Deviation scores for age-matched healthy control subjects and patients.

\begin{tabular}{lllll}
\hline & $\begin{array}{l}\text { Control } \\
\text { mean rho }\end{array}$ & $\begin{array}{l}\text { Control } \\
\text { SE }\end{array}$ & \multicolumn{2}{c}{ Mean rho deviation } \\
\cline { 4 - 5 } & & & $\begin{array}{l}\text { (Control - } \\
\text { patient) }\end{array}$ & $\begin{array}{l}\text { Deviation } \\
\text { SE }\end{array}$ \\
\hline PERCEPTUAL ATTRIBUTES & & & \\
Balance & 0.50 & 0.04 & -0.15 & 0.05 \\
Color saturation & 0.57 & 0.04 & -0.04 & 0.05 \\
Color temperature & 0.59 & 0.04 & -0.22 & 0.08 \\
Depth & 0.54 & 0.03 & -0.09 & 0.05 \\
Simplicity & 0.71 & 0.03 & -0.10 & 0.04 \\
Stroke* & 0.66 & 0.05 & -0.30 & 0.09 \\
CONCEPTUAL ATTRIBUTES & & & \\
Abstractness* & 0.81 & 0.02 & -0.25 & 0.07 \\
Animacy & 0.71 & 0.03 & -0.11 & 0.05 \\
Emotion & 0.61 & 0.03 & -0.05 & 0.04 \\
Objective accuracy* & 0.76 & 0.02 & -0.33 & 0.10 \\
Realism & 0.77 & 0.02 & -0.11 & 0.04 \\
Symbolism & 0.69 & 0.02 & -0.22 & 0.08 \\
Interest & 0.51 & 0.04 & -0.16 & 0.06 \\
Preference & 0.47 & 0.04 & -0.02 & 0.05 \\
\hline & & & & \\
\hline * & & & & \\
\hline
\end{tabular}

*denotes attributes in which deviation of patients is significantly different at $p<0.003$.

specific attribute and would show group effects when compared to control subjects. Because floor performances would mean relatively little variance in performance, that behavior would probably not correlate with variance in lesion locations in a VLSM analysis. In such a scenario, the attribute is likely to be associated with brain regions in a non-linear manner, sensitive to disruption in different possible areas. The converse is also possible. For example, if right inferior parietal damage were critical in apprehending a specific attribute, participants with such damage would have impaired performances and participants sparing this location would not. As a group, the right hemisphere damage group might not be statistically different than control participants, because many of the patients perform normally. However, in this case, VLSM analysis would reveal specific brain-behavior relationships.

For the first analysis to investigate whether right hemisphere damage in general produced specific impairments in esthetic impairment we conducted $t$-tests to test whether the group deviations were significantly different than the mean scores of the control participants, controlling for multiple comparisons at a significance level of $(p<0.003)$. We found that the patients as a group were impaired in judging the content-conceptual attributes of abstractness and depictive accuracy and the formal-perceptual attribute of stroke quality.

For the second analysis to test whether specific locations within the right hemisphere when damaged are likely to produce impairments in esthetic perception, we took the distribution of deviation scores and assess if the difference scores for each attribute obtained correlated with location of brain damage (Table 4) with a false discovery rate of 0.01 (Figure 2). In regards to the content-conceptual attributes, damage to the right frontal lobe, especially the inferior frontal gyrus, was associated with deviations in judgments of abstractness, realism, and animacy. Deviation in symbolism was associated with damage to posterolateral temporal cortex, especially the superior temporal gyrus. In addition, damage to the right parietal lobe was related to deviations in judgments of animacy. For the formal-perceptual attributes, damage to regions of the right temporal and frontal lobes as well as right insula was associated with deviations in perception of depth. Deviations in judgments of interest and preference were not associated with any specific regions of damage.

\section{DISCUSSION}

Our study examined how brain damage affects the perception and evaluation of art. We were motivated to demonstrate that quantitative approaches in the neuropsychology of art are feasible. Our study is only a first step in this direction. In what follows, we shall mention the advantages of this approach and outline our results. We then discuss some limits of the study, and how the field might move forward.

The results demonstrate that the neuropsychology of art can be investigated in a systematic and quantitative manner. We have shown previously that art production can be approached quantitatively (Smith et al., 2011). Now, we extend this approach to art perception. Quantitative approaches have the advantage of allowing formal tests of hypotheses and replication. These advantages do not denigrate the qualitative insights one might derive from careful observation and theoretical analyses of art. However, it is hard to see how the neuropsychology of art could mature as a science without quantification (Chatterjee, 2009).

Our study incorporates quantification in two ways. First, is the use of the AAA. This assessment allows quantification of specific attributes of any artwork (Chatterjee et al., 2010). There is nothing about the assessment that restricts its use to neuropsychology. The assessment could just as easily be used for other purposes, such as to compare the work of different artists or to assess the nature of change in any given artists' style over time. Second, is our use of VLSM techniques (Bates et al., 2003). This method represents a general advance in lesion analyses and is being applied to the perception of art for the first time.

Our basic findings are that damage to the right hemisphere can affect the perception of selective aspects of art (see Table 4). This cohort of patients as a group had impaired performance when judging the content-conceptual attributes of abstractness and depictive accuracy and the formal-perceptual attribute of stroke quality. We also found that damage to lateral frontal-parietaltemporal cortices was associated with deviations in the judgment of 4/6 content-conceptual art attributes: abstractness, symbolism, realism, and animacy. Of the formal-perceptual attributes, only depth was correlated with damage to the inferior prefrontal cortex. The fact that the patients as a group were impaired in judging depictive accuracy and stroke quality and these attributes did not show specific brain-behavior correlations, suggests the following hypothesis. Judging the attributes of depictive accuracy and stroke quality maybe especially vulnerable to right brain damage in different locations and these attributes may instantiated nonlinearly in the brain. No brain area was associated with deviations 


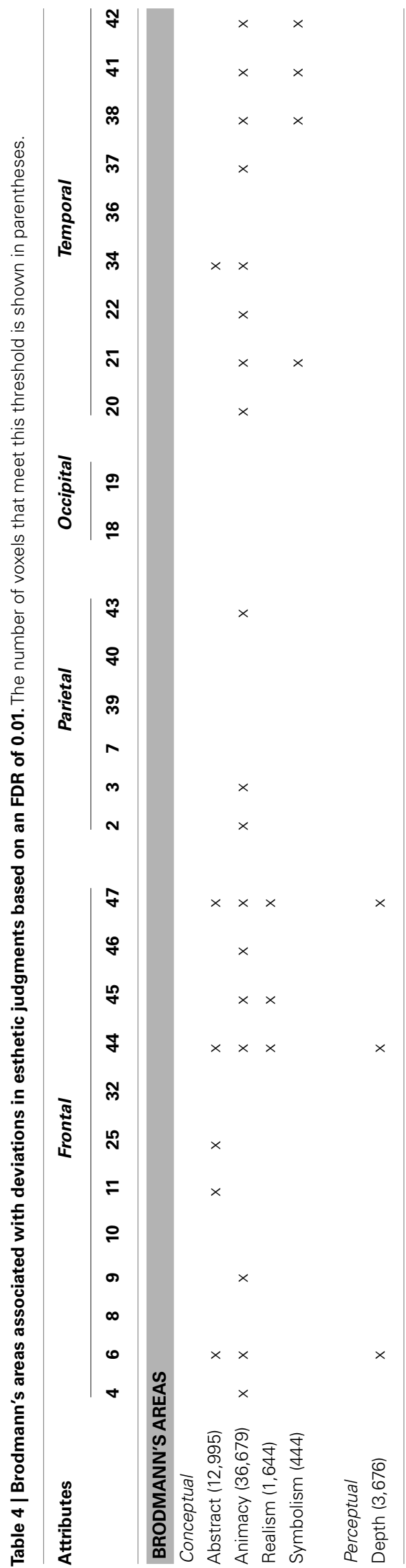

in judgments in evaluating preference or interestingness of these artworks.

Elsewhere, we have argued that any esthetic experience is built upon at least three components (Chatterjee, 2004a, 2011). These components are the experiences of the sensory qualities, the associated sets of meanings, and the emotional responses evoked by the esthetic object. Broadly, one might regard the formalperceptual attributes of the AAA as probing the sensory experience, the content-conceptual attributes as probing the meaning, and the evaluative questions as probing the emotional response to these paintings. From our data, we would tentatively propose that these three components of visual esthetic experiences segregate broadly in the organization of the brain. Most of our participants had damage in the distribution of the right middle cerebral artery. This distribution of brain damage involving lateral frontal, parietal, and temporal cortices was more likely to affect judgments of conceptual attributes. We would predict that damage in the posterior cerebral artery distribution affecting ventral occipital and temporal cortices might be more likely to affect perceptual attributes. Furthermore, given the extensive data implicating the ventral striatum and orbitofrontal cortex in assigning subjective reward values (Kable and Glimcher, 2009), we would predict that damage to ventro-medial prefrontal cortices would be more likely to affect people's evaluation of paintings.

We should be clear about the limits of this study. A general limit is that we have relatively little experimental control over ways that broad cultural and sociological factors might contribute to how people apprehend art. One would expect that cultural factors would be more likely to produce variance in judgments of content-conceptual attributes than formal-perceptual attributes (Chatterjee, 2002). Yet, w note that the content-conceptual attributes were more likely to be disrupted than the formal-perceptual attributes in this study, suggesting that role of cultural factors in this assessment were not sufficient to obscure the effects of brain damage. However, future studies that address both cultural and biological variables will be needed to provide a rich understanding of art apprehension.

Another specific limit of this study is the sampling of brain regions. While 20 participants is a relatively large group of braindamaged subjects, as mentioned above, we did not sample the ventral occipito-temporal or ventro-medial frontal cortices. We have studies currently underway to probe these areas. Another limit is that we restricted ourselves to people with right brain damage. Since this is the first study of its kind, we did not wish to confound our results with concomitant language comprehension deficits that follow from left brain damage. However, we have shown that art production can be profoundly affected by left brain damage (Smith et al., 2011). Given that production and perception must overlap at some representational levels, we would predict that left brain damage would also affect art perception. Again future studies will need to sort out the role of the left hemisphere in art perception.

Finally, we recognize that the description and evaluation of art are qualitatively different. People are more likely to agree about whether or not an image has warm tones than to agree about whether or not the image is appealing. This difference is 


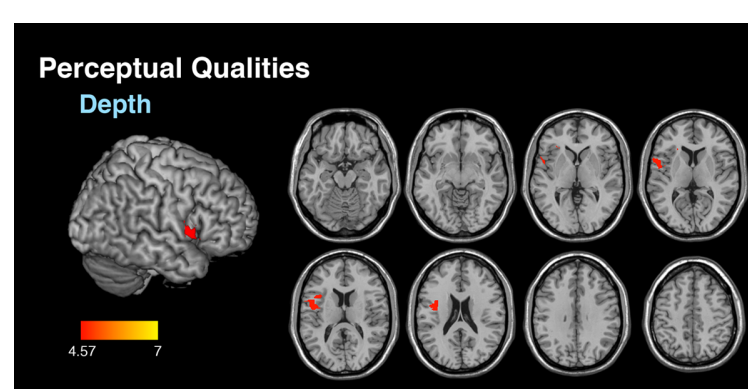

Conceptual Qualities

Abstract

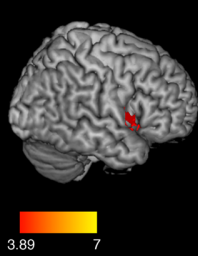

3.89
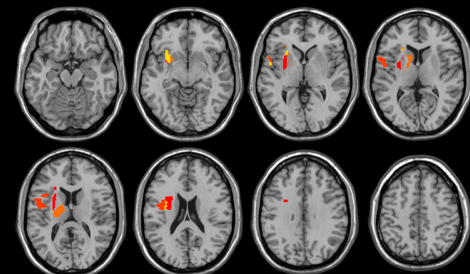

Realism
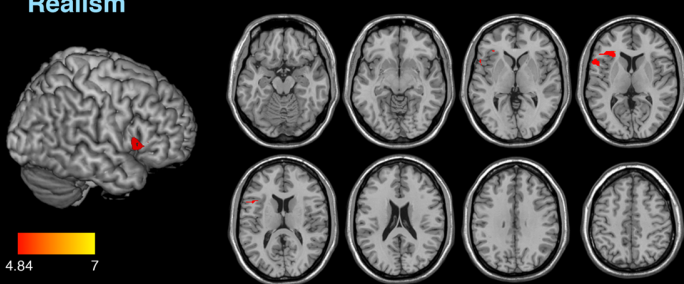
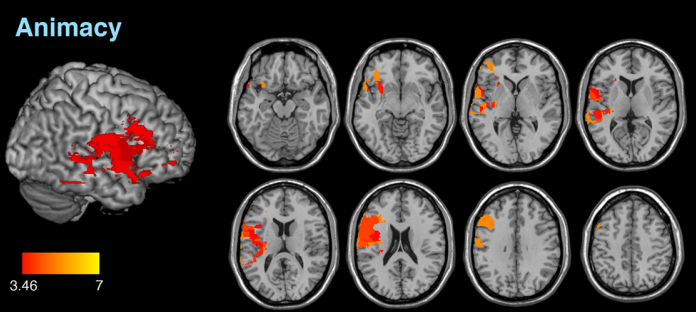

Symbolism
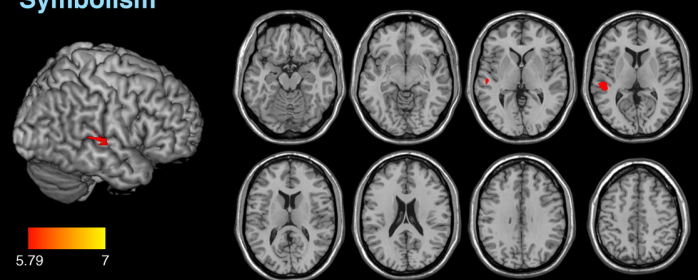

FIGURE 2 | Results of the voxel lesion symptom mapping analyses showing areas where damage was associated with significant deviations of aesthetic attribute judgments.

evident even in our healthy participants in whom agreement on preference was the lowest than it was for any of the descriptive scales. Given that the evaluation of artwork is less stable than descriptive judgments, assessing the effects of brain damage in preference is also more difficult. The problem of separating variance inherent in individual differences from those produced by the effects of brain damage remains a methodological challenge.

\section{REFERENCES}

Aharon, I., Etcoff, N., Ariely, D., Chabris, C., O'Connor, E., and Breiter, H. (2001). Beautiful faces have variable reward value: fMRI and behavioral evidence. Neuron 32, 537-551.

Alajouanine, T. (1948). Aphasia and artistic realization. Brain 71, 229-241.

Bates, E., Wilson, S., Saygin, A., Dick, F., Sereno, M., Knight, R., and Dronkers, N. (2003). Voxel-based lesion symptom mapping. Nat. Neurosci. 6, 448-450.
Bogousslavsky, J., and Boller, F. (2005). Neurological Disorders in Famous Artists, Basel: Karger.

Brown, S., Gao, X., Tisdelle, L., Eickhoff, S. B., and Liotti, M. (2011). Naturalizing aesthetics: brain areas for aesthetic appraisal across sensory modalities. Neuroimage 58, 250-258.

Cela-Conde, C. J., Ayala, F. J., Munar, E., Maestu, F., Nadal, M., Capo, M. A., del Rio, D., Lopez-Ibor, J. J., Ortiz, T. S., Mirasso, C., and Marty, G. 1. (2009). Sex-related similarities and differences in the neural correlates of

To summarize, we believe that neuropsychology will play an important role in advancing neuroesthetics. However, to date most neuropsychological reports related to art have been anecdotal and qualitative in nature. For this field to mature as a science, we advocate the use of quantitative methods. Here, we offer one approach that uses both quantitative behavioral and lesion analyses examining the role of the right hemisphere in art perception and evaluation.

beauty. Proc. Natl. Acad. Sci. U.S.A 106, 3847-3852.

Chatteriee, A. (2002). Universal and relative aesthetics: a framework from cognitive neuroscience. International Association of Empirical Aesthetics, Takarazuka, 289-292.

Chatterjee, A. (2003). "Neglect. A disorder of spatial attention," in Neurological Foundations of Cognitive Neuroscience, ed. M. D'Esposito (Cambridge, MA: The MIT Press), 1-26.

Chatterjee, A. (2004a). Prospects for a cognitive neuroscience of visual aesthetics. Bull. Psychol. Arts 4, 55-59.
Chatterjee, A. (2004b). The neuropsychology of visual artists. Neuropsychologia 42, 1568-1583.

Chatterjee, A. (2005). A madness to the methods in cognitive neuroscience? J. Cogn. Neurosci. 17, 847-849.

Chatterjee, A. (2006). The neuropsychology of visual art: conferring capacity. Int. Rev. Neurobiol. 74, 39-49.

Chatterjee, A. (2009). "Prospects for a neuropsychology of art," in Neuroaesthetics, eds M. Skov and O. Vartanian (Amityville, NY: Baywood Publishing Company), 131-143. 
Chatterjee, A. (2011). Neuroaesthetics: a coming of age story. J. Cogn. Neurosci. 23, 53-62.

Chatterjee, A., Thomas, A., Smith, S. E., and Aguirre, G. K. (2009). The neural response to facial attractiveness. Neuropsychology 23, 135-143.

Chatterjee, A., Widick, P., Sternschein, R., Smith, W. B. II, and Bromberger, B. (2010). The assessment of art attributes. Empir. Stud. Arts 28, 207-222.

Cupchik, G. C., and Gebotys, R. (1988). The experience of time, pleasure, and interest during aesthetic episodes. Empir. Stud. Arts 6, 1-12.

Fechner, G. (1876). Vorschule der Aesthetik. Leipzig: Breitkopf \& Hartel.

Fellows, L. K., Heberlein, A. S., Morales, D. A., Shivde, G., Waller, S., and Wu, D. H. (2006). Method matters: an empirical study of impact in cognitive neuroscience. J. Cogn. Neurosci. 17, 850-858.

Heilman, K. M., Watson, R. T., and Valenstein, E. (1993). "Neglect and related disorders," in Clinical Neuropsychology, eds K. M. Heilman and E. Valenstein (New York: Oxford University Press), 279-336.

Hekkert, P., and Van Wieringen, P. C. W. (1996). Beauty in the eye of expert and nonexpert beholders: a study in the appraisal of art. Am. J. Psychol. 109, 389-407.

Ishai, A. (2007). Sex, beauty and the orbitofrontal cortex. Int. J. Psychophysiol. 63, 181-185.

Ishai, A., Fairhall, S., and Pepperell, R. (2007). Perception, memory and aesthetics of indeterminate art. Brain Res. Bull. 73, 319-324.
Jacobsen, T., Schubotz, R., Hofel, L., and Cramon, D. Y. (2005). Brain correlates of aesthetic judgments of beauty. Neuroimage 29, 276-285.

Kable, J. W., and Glimcher, P. W. (2009). The neurobiology of decision: consensus and controversy. Neuron 63, 733-745.

Kawabata, H., and Zeki, S. (2004). Neural correlates of beauty. J. Neurophysiol. 91, 1699-1705.

Kimberg, D. Y., Coslett, H. B., and Schwartz, M. F. (2007). Power in voxel-based lesion-symptom mapping. J. Cogn. Neurosci. 19, 1067-1080.

Leder, H., Belke, B., Oeberst, A. and Augustin, D. (2004). A model of aesthetic appreciation and aesthetic judgments. Br. J. Psychol. 95, 489-508.

Livingstone, M. (2002). Vision and Art: The Biology of Seeing. New York: Abrams.

Mattingley, J., Berberovic, N., Corben, L., Slavin, M., Nicholls, M., and Bradshaw, J. (2004). The greyscales task: a perceptual measure of attentional bias following unilateral hemispheric damage. Neuropsychologia 42, 387-394.

Nadal, M., Munar, E., Capo, M. A., Rosselo, J., and Cela-Conde, C. J. (2008). Towards a framework for the study of the neural correlates of aesthetic preference. Spat. Vis. 21, 379-396.

Nadal, M., and Pearce, M. T. (2011). The Copenhagen neuroaesthetics conference: prospects and pitfalls for an emerging field. Brain Cogn. 76, 172-183.

Ramachandran, V. S., and Hirstein, H. (1999). The science of art: a neurological theory of aesthetic experience. J. Conscious. Stud. 6, 15-51.

Rorden, C., and Karnath, H.-O. (2004) Using human brain lesions to infer function: a relic from a past era in the fMRI age? Nat. Rev. Neurosci. 5 , 813-819.

Russell, P. A., and George, D. A. (1990). Relationships between aesthetic response scales applied to paintings. Empir. Stud. Arts 8, 15-30.

Skov, M., and Vartanian, O. (eds). (2009). Neuroaesthetics. Amityville, NY: Baywood Publishing Company, Inc.

Smith, W. B. II, Sternschein, R., Widick, P., Bromberger, B., and Chatterjee, A. (2011). Artistic production following brain damage. Leonardo 44 405-410.

van Buren, B., Bromberger, B., Potts, D., Miller, B., and Chatterjee, A. (2010). "Changes in painting styles of artists with Alzheimer's disease," in International Association of Empirical Aesthetics Annual Meeting, Dresden.

Vartanian, O., and Goel, V. (2004). Neuroanatomical correlates of aesthetic preference for paintings. Neuroreport 15, 893-897.

Warrington, E. K., and James, M. (1991). The Visual Object and Spatial Perception Battery. Bury St Edmunds: Thames Valley Test Company.

Winston, J., O’Doherty, J., Kilner, J., Perrett, D., and Dolan, R. (2007).
Brain systems for assessing facia attractiveness. Neuropsychologia 45 , 195-206.

Woods, W. A. (1991). Parameters of aesthetic objects: applied aesthetics. Empir. Stud. Arts 9, 105-114.

Wu, D. H., Waller, S., and Chatterjee, A. (2007). The functional neuroanatomy of thematic role and locative relational knowledge. $J$. Cogn. Neurosci. 19, 1542-1555.

Zaidel, D. (2005). Neuropsychology of Art. New York: Psychology Press.

Zeki, S. (1999). Art and the brain. J. Conscious. Stud. 6, 76-96.

Conflict of Interest Statement: The authors declare that the research was conducted in the absence of any commercial or financial relationships that could be construed as a potential conflict of interest.

Received: 09 March 2011; accepted: 14 September 2011; published online: 14 October 2011.

Citation: Bromberger B, Sternschein R, Widick P, Smith W II and Chatterjee A (2011) The right hemisphere in esthetic perception. Front. Hum. Neurosci. 5:109. doi: 10.3389/fnhum.2011.00109

Copyright (C) 2011 Bromberger, Sternschein, Widick, Smith and Chatterjee. This is an open-access article subject to a non-exclusive license between the authors and Frontiers Media SA, which permits use, distribution and reproduction in other forums, provided the original authors and source are credited and other Frontiers conditions are complied with. 